\title{
Composite Adaptive Fuzzy Prescribed Performance Control of Nonlinear Systems
}

\author{
Xudong Cao, ${ }^{1}$ Jianjun Wang, ${ }^{1}$ and Wei Xiang $\mathbb{D}^{2}$ \\ ${ }^{1}$ School of Highway, Chang'an University, Xi an 710064, China \\ ${ }^{2}$ Department of Mathematics, Huainan Normal University, Huainan 232038, China
}

Correspondence should be addressed to Wei Xiang; xiangwei27@126.com

Received 12 December 2019; Revised 15 February 2020; Accepted 24 February 2020; Published 26 March 2020

Guest Editor: Lazaros Moysis

Copyright (c) 2020 Xudong Cao et al. This is an open access article distributed under the Creative Commons Attribution License, which permits unrestricted use, distribution, and reproduction in any medium, provided the original work is properly cited.

This paper proposes an adaptive fuzzy prescribed performance control (PPC) method of a class of uncertain nonlinear systems. Different from the traditional PPC approach that requires the exact values of the initial conditions, by using a new type of performance function, the proposed PPC scheme together with a composite adaptation law works effectively even without the knowledge of initial conditions. Meanwhile, the constructed disturbance observer and fuzzy logic systems can estimate system uncertainties including external disturbances and fuzzy approximation errors. Under the proposed tracking controller, the boundedness of all involved signals is guaranteed, and the tracking errors satisfy the prescribed performance bounds all the time. Finally, simulation results show the efficacy of the proposed method.

\section{Introduction}

It is well known that the control performance of traditional control methods may not be satisfactory for uncertainties and external disturbances in real systems. In fact, most realworld systems' model is very hard to be achieved, and it is very hard to implement controllers for these systems. Since the fuzzy logic system can be used to model nonlinear dynamics, it can be used to deal with the uncertainty of the system. At present, adaptive fuzzy control for uncertain systems has been widely studied [1-9]. Adaptive fuzzy control method is effective for tackling parametric uncertainties and external disturbances, in which tracking errors are utilized to update fuzzy parameters as well as to approximate unknown nonlinear functions. For fractionalorder modified chaotic systems, linear control and fuzzy control are combined to realize synchronous control in [10]. Adaptive fuzzy impulsive control for nonlinear systems was presented in [11]. On the contrary, prescribed performance control (PPC) methods are proposed for nonlinear systems to get rapid stability of the controlled systems [12-16]. The PPC method usually includes two aspects: one is transforming the constrained system into an unconstrained equivalent transformation system, and the other is to prove that the transformed variable remains bounded. For example, in [13], PPC and backstepping technique were combined to explore a class of nontriangular structure nonlinear systems. Shao and Tong [14] investigated an effective PPC scheme for MIMO stochastic nonlinear systems. Usually, the traditional prescribed performance boundary (PPB) of tracking error $e$ is described as

$$
\begin{gathered}
-\lambda \mu(t)<e<\mu(t), \quad \text { if } e(0)>0, \\
-\mu(t)<e<\lambda \mu(t), \quad \text { if } e(0)<0,
\end{gathered}
$$

where $\lambda \in[0,1], \mu(t)=\left(\lambda_{0}-\lambda_{\infty}\right) e^{-\lambda_{1} t}+\lambda_{\infty}$, and $\lambda_{0}, \lambda_{\infty}$, and $\lambda_{1}$ are design parameters. If the initial value $e(0)$ is unknown, the controller of PPC may not meet PPB (1), resulting in poor control performance. Recently, $\mathrm{Bu}$ et al. [17] proposed prescribed performance neural controllers of a flexible airbreathing hypersonic vehicle. By using the property of performance function, the problem of unknown initial value was solved, and the prescribed performance of the system state is guaranteed. Therefore, the performance function in [17] will be used to investigate the uncertain nonlinear system with dead-zone inputs. 
Learning ability is a fundamental feature of all intelligent behaviors. Up to now, there are many learning control methods which have been proposed for nonlinear systems. Iterative learning control technology is applicable to the repeated operation system on finite time under the premise that the initial value of the iteration error is zero [18-20]. After enough iterations, the controller with reasonable design parameters can realize the zero error of the system output/state over the entire operation interval. However, due to the limitation of the reset condition, there is an initial error in the actual situation. For various nonlinear uncertainties including the parameter uncertain system and the nonparametric uncertain system, designing learning controller based on Lyapunov synthesis method, solving the corresponding trajectory tracking problem, is a research hotspot in the field of learning control. To solve the problem of nonzero error initial value and to design the learning control system, the composite learning control method is proposed by Pan et al. [21, 22]. Based on comprehensive learning techniques, an adaptive dynamic surface control algorithm was proposed for integerorder systems with mismatched parameters in [21]. The interval integral of online recorded data was used to generate prediction error and instantaneous tracking error to update the parameter estimation. The composite learning technology can solve the shortcomings of the parallel learning adaptive control algorithm and effectively realize the parameter convergence under the condition of continuous excitation in the integer-order system. In [23, 24], a disturbance observer was proposed for nonlinear systems by means of the adaptive fuzzy composite learning control, where the PPC problem is not considered. In $[25,26]$, composite learning control was extended for fractional-order systems. To our knowledge, the composite learning PPC is rarely investigated.

Inspired by the above works, this paper researches adaptive fuzzy PPC for nonlinear systems with unknown initial conditions. Firstly, the PPB conditions are given by the performance function. Then, the original system is transformed into an equivalent transformation system. Afterwards, an external disturbance observer is designed, which can estimate system uncertainties including external disturbances and fuzzy approximation errors. The proposed adaptive fuzzy composite learning law can not only guarantee the prescribed performance but also make accurate estimates of system uncertainties. The main contributions of this paper are summarized as follows. (1) The control method proposed in this paper can overcome the dependence of traditional PPC on initial values. (2) Disturbance observers constructed with prediction errors can accurately estimate system uncertainties. (3) Compared with the traditional adaptive fuzzy PPC method, the proposed method has better control performance.

The rest of this work are arranged as follows. Some basic mathematical results and the problem statement are presented in Section 2. The prescribed performance transformation, the adaptive fuzzy PPC design, and the observer design, as well as the stability analysis are included in Section 3. A simulation example is presented in Section 4 to show the validity of the proposed method, and some comparison results are also given. At last, Section 5 gives a sum-up of the whole research.

\section{Preliminaries}

Consider the following nonlinear system with dead-zone inputs

$$
\dot{\mathbf{x}}=\mathbf{f}(\mathbf{x})+\mathbf{d}(\mathbf{t})+\mathbf{G}(\mathbf{x}) \Gamma(\mathbf{u})
$$

where $\mathbf{x}=\left[x_{1}, x_{2}, \ldots, x_{n}\right]^{T} \in R^{n}$ is the state vector which is assumed to be measurable, $\mathbf{f}(\mathbf{x})=\left[f_{1}(\mathbf{x}), f_{2}(\mathbf{x}), \ldots\right.$, $\left.f_{n}(\mathbf{x})\right]^{T}$ is an unknown smooth function, $\mathbf{G}(\mathbf{x})=\operatorname{diag}\left(g_{1}(\mathbf{x})\right.$, $\left.g_{2}(\mathbf{x}), \ldots, g_{n}(\mathbf{x})\right)$ is a coefficient matrix, and $g_{i}(\mathbf{x})$ is a known function, $i=1,2, \ldots, n . \mathbf{d}(\mathbf{t})=\left[d_{1}(t), d_{2}(t), \ldots\right.$, $\left.d_{n}(t)\right]^{T}$ is an unknown external disturbance. $\mathbf{u}=\left[u_{1}, u_{2}, \ldots\right.$, $\left.u_{n}\right]^{T}$ is the controller output vector, and $\boldsymbol{\Gamma}(\mathbf{u})=\left[\boldsymbol{\Gamma}_{1}\left(u_{1}\right)\right.$, $\left.\Gamma_{2}\left(u_{2}\right), \ldots, \Gamma_{n}\left(u_{n}\right)\right]^{T}$ is the controller input vector subject to dead-zone type nonlinearity. $\Gamma_{i}\left(u_{i}\right)$ is described as

$$
\Gamma_{i}\left(u_{i}\right)= \begin{cases}m\left(u_{i}-b_{r i}\right), & \text { if } u_{i} \geq b_{r i}, \\ 0, & \text { if }-b_{l i}<u_{i}<b_{r i}, \\ m\left(u_{i}+b_{l i}\right), & \text { if } u_{i} \leq-b_{l i},\end{cases}
$$

where $m, b_{r i}$, and $b_{l i}$ are positive constants. So, one can write (3) as

$$
\Gamma_{i}\left(u_{i}\right)=m u_{i}+\Delta u_{i}
$$

where

$$
\Delta u_{i}= \begin{cases}-m b_{i r}, & \text { if } u_{i} \geq b_{i r}, \\ -m u_{i}, & \text { if }-b_{i l}<u_{i}<b_{i r}, \\ m b_{i l}, & \text { if } u_{i} \leq-b_{i l} .\end{cases}
$$

According to (4), system (1) can be rewritten as

$$
\dot{\mathbf{x}}=\mathbf{f}(\mathbf{x})+m \mathbf{G}(\mathbf{x}) \mathbf{u}+\overline{\mathbf{d}}(\mathbf{t}),
$$

where $\overline{\mathbf{d}}(\mathbf{t})=\left[\bar{d}_{1}(t), \bar{d}_{2}(t), \ldots, \bar{d}_{n}(t)\right]^{T}$ and $\bar{d}_{i}(t)=d_{i}(t)+$ $\Delta u_{i}$.

Define $\mathbf{e}=\mathbf{x}-\mathbf{x}_{d}=\left[e_{1}, e_{2}, \ldots, e_{n}\right]^{T}$ and $e_{i}=x_{i}-x_{d i}$, and $x_{d i}$ is the reference signal, $i=1,2, \ldots, n$. The following assumptions need to be given:

Assumption 1. The reference signal $x_{d i}$ and $\dot{x}_{d i}$ are known, and the initial $x_{i}(0)$ is unknown but bounded Assumption 2. The nonlinear function $f_{i}(\mathbf{x})$ is unknown but bounded

Assumption 3. The gain function $g_{i}(\mathbf{x})$ is known and $g_{i}(\mathbf{x}) \neq 0$ for all $\mathbf{x}$

Assumption 4. The unknown external disturbance $d_{i}(t)$ is time varying and satisfies $\left|d_{i}(t)\right| \leq l_{1 i}$ and $\left|\dot{d}_{i}(t)\right| \leq l_{2 i}$, where $l_{1 i}$ and $l_{2 i}$ are unknown positive constants

Remark 1. It is well known that, in real-world systems, the initial conditions are very hard to be obtained, i.e., the initial conditions are unknown. In this paper, to solve this problem, based on above assumptions, this paper will design an adaptive fuzzy prescribed performance controller.

Remark 2. In this paper, $f_{i}(\overline{\mathbf{x}})$ is assumed unknown smooth function, so fuzzy logic systems (FLSs) will be used to approximate it. If $g_{i}(\overline{\mathbf{x}})$ is an uncertain smooth nonzero 
function, one can divide $g_{i}(\overline{\mathbf{x}})$ into the nominal part $\bar{g}_{i}\left(\overline{\mathbf{x}}_{i}\right)$ and the perturbed part $\Delta g_{i}$ and incorporate $\Delta g_{i}$ into $f_{i}(\overline{\mathbf{x}})$ and define $\bar{f}_{i}(\overline{\mathbf{x}})$ as

$$
\bar{f}_{i}(\overline{\mathbf{x}})=f_{i}(\overline{\mathbf{x}})+\Delta g_{i} \Gamma_{i}\left(u_{i}\right),
$$

and then estimate $\bar{f}_{i}(\overline{\mathbf{x}})$ by using FLSs. Therefore, this paper only considers the case that $g_{i}(\overline{\mathbf{x}})$ is known smooth nonzero function.

Here, define $F_{i}(\mathbf{x})=l_{i} f_{i}(\mathbf{x})$, where $l_{i}$ is a positive design constant and $\widehat{F}_{i}(\mathbf{x})=\widehat{\theta}_{F_{i}}^{T} \varphi_{F_{i}}(x)$ is the approximate of $F_{i}(\mathbf{x})$ by using FLSs, where $\widehat{\vartheta}_{F_{i}}$ is an adjustable parameter vector and $\varphi_{F_{i}}(\mathbf{x})$ is the fuzzy basis function vector. And $F_{i}(\mathbf{x})=\theta_{F_{i}}^{* T} \varphi_{F_{i}}(\mathbf{x})+\varepsilon_{F_{i}}(\mathbf{x}), \quad \theta_{F_{i}}^{*}$ is the optimal parameter vector, and $\varepsilon_{F_{1}}(\mathbf{x})$ is the approximate error. Defining $\widetilde{\theta}=\theta^{*}-\widehat{\theta}$, one has

$$
\begin{aligned}
f_{i}(\mathbf{x}) & =l_{i}^{-1} F_{i}(\mathbf{x}) \\
& =l_{i}^{-1} \theta_{F_{i}}^{* T} \varphi_{F_{i}}(\mathbf{x})+l_{i}^{-1} \varepsilon_{F_{i}}(\mathbf{x}) \\
& =l_{i}^{-1}\left(\tilde{\theta}_{F_{i}}^{T}+\tilde{\theta}_{F_{i}}^{T}\right) \varphi_{F_{i}}(\mathbf{x})+l_{i}^{-1} \varepsilon_{F_{i}}(\mathbf{x}) \\
& =l_{i}^{-1} \widetilde{\theta}_{F_{i}}^{T} \varphi_{F_{i}}(\mathbf{x})+l_{i}^{-1} \widehat{\theta}_{F_{i}}^{T} \varphi_{F_{i}}(\mathbf{x})+l_{i}^{-1} \varepsilon_{F_{i}}(\mathbf{x}) .
\end{aligned}
$$

Let $\quad$ us denote $\quad \mathbf{L}^{-1} \tilde{\theta}_{F}^{T} \quad \varphi_{F}(\mathbf{x})=\left[l_{1}^{-1} \tilde{\theta}_{F_{1}}^{T} \varphi_{F_{1}}(\mathbf{x}), l_{2}^{-1} \tilde{\theta}_{F_{2}}^{T}\right.$ $\left.\varphi_{F_{2}}(\mathbf{x}), \ldots, l_{n}^{-1} \widetilde{\theta}_{F_{n}}^{T} \varphi_{F_{n}}(\mathbf{x})\right]^{T}, \quad \mathbf{L}^{-1} \widehat{\theta}_{F}^{T} \varphi_{F}(\mathbf{x})=\left[l_{1}^{-1} \widehat{\theta}_{F_{1}}^{T} \varphi_{F_{1}}(\mathbf{x}), l_{2}^{-1}\right.$ $\left.\widehat{\theta}_{F_{2}}^{T} \varphi_{F_{2}}(\mathbf{x}), \ldots, l_{n}^{-1} \widehat{\theta}_{F_{n}}^{T} \varphi_{F_{n}}(\mathbf{x})\right]^{T}$, and $L^{-1} \varepsilon_{F}(\mathbf{x})=\left[l_{1}^{-1} \varepsilon_{F_{1}}(\mathbf{x}), l_{2}^{-1}\right.$ $\left.\varepsilon_{F_{2}}(\mathbf{x}), \ldots, l_{n}^{-1} \varepsilon_{F_{n}}(\mathbf{x})\right]^{T}$. So, $\mathbf{f}(\mathbf{x})$ in (6) can be expressed as

$$
\mathbf{f}(\mathbf{x})=\mathbf{L}^{-1} \widetilde{\theta}_{F}^{T} \varphi_{F}(\mathbf{x})+\mathbf{L}^{-1} \widehat{\theta}_{F}^{T} \varphi_{F}(\mathbf{x})+\mathbf{L}^{-1} \varepsilon_{F}(\mathbf{x}) .
$$

Let $\mathbf{D}=\mathbf{L}^{-1} \varepsilon_{F}(\mathbf{x})+\overline{\mathbf{d}}(\mathbf{t})$. The error system of $e$ can be described as

$$
\dot{\mathbf{e}}=\mathbf{L}^{-1} \tilde{\theta}_{F}^{T} \varphi_{F}(\mathbf{x})+m \mathbf{G}(\mathbf{x}) \mathbf{u}+\mathbf{L}^{-1} \widehat{\theta}_{F}^{T} \varphi_{F}(\mathbf{x})+\mathbf{D}-\dot{\mathbf{x}}_{d} .
$$

Remark 3. From (10), one knows that the unknown vector $\mathbf{D}$ is composed by the external disturbance $\mathbf{d}(\mathbf{t})$, the approximate error $\varepsilon_{F}$, and $\Delta \mathbf{u}$. So, the disturbance observer $\widehat{D}$ will be constructed so that $\widehat{\mathbf{D}}+\theta_{F}^{T} \varphi_{F}(\mathbf{x})$ can estimate $\mathbf{d}(\mathbf{t})+\mathbf{f}(\mathbf{x})+$ $\Delta \mathbf{u}$ in this paper.

The aim of this paper is (i) to use a given performance function to get rid of the dependence on the sign of the initial value $e(0)$; (ii) to design the composite learning control with the disturbance observer to make sure that the tracking error $e$ satisfies the prescribed performance boundary; and (iii) to estimate system uncertainties accurately.

\section{Control Design and Stability Analysis}

3.1. Prescribed Performance. It is assumed here that the tracking error $e_{i}$ satisfies the following prescribed performance boundary (PPB) for $i=1,2, \ldots, n$ :

$$
-\underline{\lambda} y_{i}(t)<e_{i}<\bar{\lambda} y_{i}(t)
$$

where $\underline{\lambda}$ and $\bar{\lambda}$ are two adjustable positive constants and $y_{i}(t)$ is a performance function. Notice that $x_{i}(0)$ is unknown, which leads to $e_{i}(0)$ is unknown. To make sure that $-\underline{\lambda} y_{i}(0)<e_{i}(0)<\bar{\lambda} y_{i}(0)$ holds, one chooses the performance function $y_{i}(t)$ [17] as

$$
y_{i}(t)=\operatorname{coth}\left(\lambda_{1 i} t+\lambda_{2 i}\right)-1+\lambda_{\infty},
$$

where $\lambda_{1 i}, \lambda_{2 i}$, and $\lambda_{\infty}$ are designed positive constants. Obviously, $y_{i}(t)$ has the following properties:

(1) $y_{i}(t)$ is positive and decreasing

(2) $y_{i}(0)=\operatorname{coth}\left(\lambda_{2 i}\right)-1+\lambda_{\infty}=\left(e^{2 \lambda_{2 i}}+1 / e^{2 \lambda_{2 i}}-1\right)-1+$ $\lambda_{\infty}>\lambda_{\infty}$

(3) $\lim _{\lambda_{2 i} \longrightarrow 0^{+}} y_{i}(0)=+\infty$

(4) $\lim _{t \longrightarrow+\infty} y_{i}(t)=\lambda_{\infty}$

By using property 3 of the performance function $y_{i}(t)$, we know that $-\underline{\lambda} \lim _{\lambda_{2 i} \longrightarrow 0^{+}} y_{i}(0)=-\infty$ and $\bar{\lambda} \lim _{\lambda_{2 i} \longrightarrow 0^{+}}$ $y_{i}(0)=+\infty$. Then, for any unknown bounded initial value $e_{i}(0)$, we can select the value of $\lambda_{2 i}$ adequately small so that the following inequality holds:

$$
-\underline{\lambda} y_{i}(0)<e_{i}(0)<\bar{\lambda} y_{i}(0) \text {. }
$$

Next, an error transformation function $S\left(z_{i}\right)$ will be introduced to transform $e_{i}$ into a transformation error signal $z_{i}$. Let

$$
e_{i}=y_{i}(t) S\left(z_{i}\right)
$$

where $S\left(z_{i}\right)=\left(\bar{\lambda} e^{z_{i}}-\underline{\lambda} e^{-z_{i}} / e^{z_{i}}+e^{-z_{i}}\right)$. Apparently, $S\left(z_{i}\right)$ has the following properties:

(1) $S\left(z_{i}\right)$ is a strictly increasing smooth function

(2) $\lim _{z_{i} \longrightarrow-\infty} S\left(z_{i}\right)=-\underline{\lambda}, \lim _{z_{i} \longrightarrow+\infty} S\left(z_{i}\right)=\bar{\lambda}$

From properties 1 and 2 of $S\left(z_{i}\right)$, one knows that $-\underline{\lambda}<S\left(z_{i}\right)<\bar{\lambda}$. Multiplying both sides of the above inequality by $y_{i}(t)$, we have $-\underline{\lambda} y_{i}(t)<y_{i}(t) S\left(z_{i}\right)<\bar{\lambda} y_{i}(t)$. Therefore, one can use (14) to represent PPB (11).

Now, $y_{i}$ is denoted as $y_{i}(t)$. Because $S\left(z_{i}\right)$ is strictly increasing function, one obtains

$$
z_{i}=S^{-1}\left(z_{i}\right)=T\left(\frac{e_{i}}{y_{i}}\right)=\frac{1}{2} \ln \left(\frac{\left(e_{i} / y_{i}\right)+\underline{\lambda}}{\bar{\lambda}-\left(e_{i} / y_{i}\right)}\right),
$$

and the following transformation error system

$$
\dot{z}_{i}=r_{i}\left(\dot{e}_{i}-\frac{\dot{y}_{i}}{y_{i}} e_{i}\right)
$$

where $r_{i}=\left(1 / 2 y_{i}\right)\left(\left(1 /\left(e_{i} / y_{i}\right)+\underline{\lambda}\right)-\left(1 /\left(e_{i} / y_{i}\right)-\bar{\lambda}\right)\right)>0$ and $\dot{y}_{i}(t)=\lambda_{1 i}-\lambda_{1 i}\left[\operatorname{coth}\left(\lambda_{1 i} t+\lambda_{2 i}\right)\right]^{2}$.

Lemma 1. If $z_{i}$ is bounded, then $P P B$ (11) holds.

Proof. Since $z_{i}$ is bounded, there exists a positive constant $M$ such that $\left|z_{i}\right| \leq M$. Noting that $S_{i}\left(z_{i}\right)$ is strictly monotonic increasing, one has $-\underline{\lambda}=\lim _{M \longrightarrow+\infty} S(-M)<S(-M) \leq$ $S\left(z_{i}\right)=\left(e_{i} / y_{i}\right) \leq S(M)<\lim _{M \longrightarrow+\infty} S(M)=\underline{\lambda}$, that is, $-\underline{\lambda} y_{i}<e_{i}<\bar{\lambda} y_{i}$ holds. 
Now, according to (10) and (11), the transformation system is obtained as follows:

$\dot{\mathbf{z}}=\mathbf{r}\left(\mathbf{L}^{-1} \widehat{\theta}_{F}^{T} \varphi_{F}(\mathbf{x})+m \mathbf{G}(\mathbf{x}) \mathbf{u}+\mathbf{L}^{-1} \widetilde{\theta}_{F}^{T} \varphi_{F}(\mathbf{x})+\mathbf{D}-\dot{\mathbf{x}}_{d}-\frac{\dot{\mathbf{y}} \mathbf{y}}{\mathbf{y}}\right)$,

where $\mathbf{r}=\operatorname{diag}\left(r_{1}, r_{2}, \ldots, r_{n}\right)$ and $(\dot{\mathbf{y}} \mathbf{e} / \mathbf{y})=\left[\left(\dot{y}_{1} e_{1} / y_{1}\right)\right.$, $\left.\left(\dot{y}_{2} e_{2} / y_{2}\right), \ldots,\left(\dot{y}_{n} e_{n} / y_{n}\right)\right]^{T}$. In order to show the performance of FLSs, $\widehat{\mathbf{z}}$ is introduced as the estimation of $\mathbf{z}$, and

$$
\dot{\hat{\mathbf{z}}}=\mathbf{r}\left(-\mathbf{K} \widetilde{\mathbf{z}}+\mathbf{L}^{-1} \hat{\theta}_{F}^{T} \varphi_{F}(\mathbf{x})+m \mathbf{G}(\mathbf{x}) \mathbf{u}+\widehat{\mathbf{D}}-\dot{\mathbf{x}}_{d}-\frac{\dot{\mathbf{y}} \mathbf{e}}{\mathbf{y}}\right),
$$

where $\widetilde{\mathbf{z}}=\widehat{\mathbf{z}}-\mathbf{z}$ and $\mathbf{K}=\operatorname{diag}\left(k_{1}, k_{2}, \ldots, k_{n}\right), k_{i}$ is the positive design constant.

The disturbance observer $\widehat{\mathbf{D}}$ is constructed as

$$
\left\{\begin{array}{l}
\widehat{\mathbf{D}}=l_{0}(\mathbf{z}-\mathbf{v}), \\
\dot{\mathbf{v}}=\mathbf{r}\left(\mathbf{L}^{-1} \widehat{\theta}_{F}^{T} \varphi_{F}(\mathbf{x})+m \mathbf{G}(\mathbf{x}) \mathbf{u}+\widehat{\mathbf{D}}-\dot{\mathbf{x}}_{d} \frac{-\dot{\mathbf{y}} \mathbf{e}}{\mathbf{y}}+l_{0}^{-1}(-\mathbf{z}+\Pi \widetilde{\mathbf{z}})\right),
\end{array}\right.
$$

where $\Pi=\operatorname{diag}\left(\pi_{1}, \pi_{2}, \ldots, \pi_{n}\right), \pi_{i}$ and $l_{0}$ are positive design parameters.

The derivative of $\widehat{\mathbf{D}}$ yields

$$
\begin{aligned}
\dot{\hat{\mathbf{D}}} & =l_{0}(\dot{\mathbf{z}}-\dot{\mathbf{v}}) \\
& =l_{0} \mathbf{r}\left(\mathbf{L}^{-1} \widetilde{\theta}_{F}^{T} \varphi_{F}(x)+\widetilde{\mathbf{D}}\right)+\mathbf{r z}-\mathbf{r} \Pi \widetilde{\mathbf{z}},
\end{aligned}
$$

where $\widetilde{\mathbf{D}}=\mathbf{D}-\widehat{\mathbf{D}}$. Furthermore, one obtains

$$
\dot{\tilde{D}}=\dot{\mathbf{D}}-\mathbf{r z}+\mathbf{r} \Pi \widetilde{\mathbf{z}}-l_{0} \mathbf{r}\left(\mathbf{L}^{-1} \tilde{\theta}_{F}^{T} \varphi_{F}(\mathbf{x})+\widetilde{\mathbf{D}}\right) .
$$

3.2. Main Result. Based on the above analysis, the main result of this paper is given.

Theorem 1. For nonlinear system (1) with unknown initial value $\boldsymbol{x}(0)$ and the time-varying external disturbance $\boldsymbol{d}(\boldsymbol{t})$, if the controller $\boldsymbol{u}(t)$ and the composite learning-based $p a-$ rameter adaptive laws are designed as

$$
\begin{aligned}
\mathbf{u} & =\frac{1}{m} \mathbf{G}^{-1}(\mathbf{x})\left[-\mathbf{C} \mathbf{z}-\mathbf{L}^{-1} \widehat{\theta}_{F} \varphi_{F}(\mathbf{x})-\widehat{\mathbf{D}}+\frac{\dot{\mathbf{y}}}{\mathbf{y}}+\dot{\mathbf{x}}_{\mathbf{d}}\right], \\
\dot{\hat{\theta}}_{F_{i}} & =\eta_{F_{i}}\left[l_{i}^{-1} z_{i} \varphi_{F_{i}}(\mathbf{x})-\pi_{i} l_{i}^{-1} \widetilde{z}_{i} \varphi_{F_{i}}(\mathbf{x})-\delta_{i} \widehat{\theta}_{F_{i}}\right],
\end{aligned}
$$

where $C=\operatorname{diag}\left(c_{1}, c_{2}, \ldots, c_{n}\right), c_{i}, \delta_{i}$, and $\eta_{F_{i}}$ are positive design constants, $i=1,2, \ldots, n$, and the disturbance observer is designed as (19), then all the close-loop system signals in (23) are uniformly ultimately bounded. Furthermore, the error state $e_{i}$ satisfies PPB (11).

Proof. Define the Lyapunov function as

$$
V=V_{1}+V_{2}+V_{3}+V_{4},
$$

where

$$
\begin{aligned}
& V_{1}=\frac{1}{2} z^{T} r^{-1} z=\sum_{i=1}^{n} \frac{1}{2} \frac{z_{i}^{2}}{r_{i}}, \\
& V_{2}=\frac{1}{2} \widetilde{D}^{T} r^{-1} \widetilde{D}=\sum_{i=1}^{n} \frac{1}{2} \frac{\widetilde{D}_{i}^{2}}{r_{i}}, \\
& V_{3}=\sum_{i=1}^{n} \frac{1}{2 \eta_{F_{i}}} \widetilde{\theta}_{F_{i}}^{T} \widetilde{\theta}_{F_{i}}, \\
& V_{4}=\frac{1}{2} \widetilde{z}^{T} r^{-1} \Pi \widetilde{z}=\sum_{i=1}^{n} \frac{1}{2} \frac{\pi_{i} \widetilde{z}_{i}^{2}}{r_{i}} .
\end{aligned}
$$
follows:

Then, the derivatives of $V_{i}, i=1,2,3,4$ can be given as

$$
\begin{aligned}
\dot{V}_{1}= & \sum_{i=1}^{n}\left[\frac{z_{i} \dot{z}_{i}}{r_{i}}-\frac{\dot{r}_{i} z_{i}^{2}}{2 r_{i}^{2}}\right] \\
= & \sum_{i=1}^{n}\left[z _ { i } \left(l_{i}^{-1} \widehat{\theta}_{F_{i}}^{T} \varphi_{F_{i}}(\mathbf{x})+m g_{i}(\mathbf{x})-\frac{\dot{y}_{i} e_{i}}{y_{i}}-\dot{x}_{d i}+D_{i}(t)\right.\right. \\
& \left.\left.+l_{i}^{-1} \widetilde{\theta}_{F_{i}}^{T} \varphi_{F_{i}}(\mathbf{x})-\frac{\dot{r}_{i} z_{i}^{2}}{2 r_{i}^{2}}\right)\right] \\
= & \sum_{i=1}^{n}\left[-\left(c_{i}+\frac{\dot{r}_{i}}{2 r_{i}^{2}}\right) z_{i}^{2}+z_{i} \widetilde{D}_{i}+l_{i}^{-1} z_{i} \widetilde{\theta}_{F_{i}}^{T} \varphi_{F_{i}}(\mathbf{x})\right],
\end{aligned}
$$

$$
\begin{aligned}
\dot{V}_{2}= & \sum_{i=1}^{n}\left[\frac{\widetilde{D}_{i} \dot{\tilde{D}}_{i}}{r_{i}}-\frac{\dot{r}_{i} \widetilde{D}_{i}^{2}}{2 r_{i}^{2}}\right] \\
= & \sum_{i=1}^{n}\left[-\left(l_{0}+\frac{\dot{r}_{i}}{2 r_{i}^{2}}\right) \widetilde{D}_{i}^{2}-\widetilde{D}_{i} z_{i}+\pi_{i} \widetilde{z}_{i} \widetilde{D}_{i}+\frac{\widetilde{D}_{i} \dot{D}_{i}}{r_{i}}\right. \\
& \left.-l_{0} l_{i}^{-1} \widetilde{D}_{i} \widetilde{\theta}_{F_{i}}^{T} \varphi_{F_{i}}(\mathbf{x})\right], \\
\dot{V}_{3}= & \sum_{i=1}^{n} \frac{1}{\eta_{F_{i}}} \widetilde{\theta}_{F_{i}}^{T} \dot{\tilde{\theta}}_{F_{i}} \\
= & \sum_{i=1}^{n}\left[-l_{i}^{-1} z_{i} \widetilde{\theta}_{F_{i}}^{T} \varphi_{F_{i}}(\mathbf{x})+\pi_{i} l_{i}^{-1} \widetilde{z}_{i} \widetilde{\theta}_{F_{i}}^{T} \varphi_{F_{i}}(\mathbf{x})+\delta_{i} \widetilde{\theta}_{F_{i}}^{T} \widehat{\theta}_{F_{i}}\right],
\end{aligned}
$$

$$
\begin{aligned}
\dot{V}_{4} & =\sum_{i=1}^{n}\left[\frac{\pi_{i} z_{i} \dot{\tilde{z}}_{i}}{r_{i}}-\frac{\pi_{i} \dot{r}_{i} \widetilde{z}_{i}^{2}}{2 r_{i}^{2}}\right] \\
& =\sum_{i=1}^{n}\left[\pi_{i} \widetilde{z}_{i}\left(-k_{i} \widetilde{z}_{i}-\widetilde{D}_{i}-l_{i}^{-1} \widetilde{\theta}_{F_{i}}^{T} \varphi_{F_{i}}(\mathbf{x})\right)+\frac{\pi_{i} \tilde{r}_{i} \widetilde{z}_{i}^{2}}{2 r_{i}^{2}}\right] \\
& =\sum_{i=1}^{n}\left[-\left(k_{i} \pi_{i}+\frac{\pi_{i} \dot{r}_{i}}{2 r_{i}^{2}}\right) \tilde{z}_{i}^{2}-\pi_{i} \widetilde{z}_{i} \widetilde{D}_{i}-\pi_{i} l_{i}^{-1} \widetilde{z}_{i} \widetilde{\theta}_{F_{i}}^{T} \varphi_{F_{i}}(\mathbf{x})\right] .
\end{aligned}
$$


Using (25)-(28), one has

$$
\begin{aligned}
\dot{V}= & \sum_{i=1}^{n}\left[-\left(c_{i}+\frac{\dot{r}_{i}}{2 r_{i}^{2}}\right) z_{i}^{2}-\left(l_{0}+\frac{\dot{r}_{i}}{2 r_{i}^{2}}\right) \widetilde{D}_{i}^{2}-\left(k_{i} \pi_{i}+\frac{\pi_{i} \dot{r}_{i}}{2 r_{i}^{2}}\right) \widetilde{z}_{i}^{2}\right. \\
& \left.-l_{0} l_{i}^{-1} \widetilde{D}_{i} \widetilde{\theta}_{F_{i}}^{T} \varphi_{F_{i}}(\mathbf{x})+\frac{\widetilde{D}_{i} \dot{D}_{i}}{r_{i}}+\delta_{i} \widetilde{\theta}_{F_{i}}^{T} \widehat{\theta}_{F_{i}}\right] .
\end{aligned}
$$

According to Meng and Moore [20], there exist unknown positive constants $\chi_{i}$ and $\varrho_{i}$ such that $\left\|\varphi_{F_{i}}(x)\right\| \leq \chi_{i}$ and $\left\|\dot{D}_{i}\right\| \leq \varphi_{i}$, respectively. So, the following inequalities hold:

$$
\begin{aligned}
\frac{\widetilde{D}_{i} \dot{D}_{i}}{r_{i}} \leq \frac{\widetilde{D} i^{2}}{2 r_{i}^{2}}+\frac{\dot{D} i^{2}}{2} \leq \frac{\widetilde{D} i^{2}}{2 r_{i}^{2}}+\frac{\varrho_{i}^{2}}{2}, \\
\widetilde{D}_{i} \widetilde{\theta}_{F_{i}}^{T} \varphi_{F_{i}}(\mathbf{x}) \leq \frac{\nu_{i} \chi_{i}^{2} \widetilde{D}_{i}^{2}}{2}+\frac{\left\|\widetilde{\theta}_{F_{i}}\right\|^{2}}{2 v_{i}}, \\
\delta_{i} \widetilde{\theta}_{F_{i}}^{T} \widehat{\theta}_{F_{i}} \leq-\frac{\delta_{i}}{2}\|\widetilde{\theta}\|^{2}+\frac{\delta_{i}}{2}\left\|\theta^{*}\right\|^{2},
\end{aligned}
$$

where $v_{i}$ is a positive constant. Substituting (30) into (29), one can obtain

$$
\begin{aligned}
\dot{V}= & \sum_{i=1}^{n}\left[-\left(c_{i}+\frac{\dot{r}_{i}}{2 r_{i}^{2}}\right) z_{i}^{2}-\left(l_{0}+\frac{\dot{r}_{i}}{2 r_{i}^{2}}-\frac{v_{i} l_{0} l^{-1} \chi_{i}^{2}}{2}\right) \widetilde{D}_{i}^{2}\right. \\
& -\left(k_{i} \pi_{i}+\frac{\pi_{i} \dot{r}_{i}}{2 r_{i}^{2}}\right) \widetilde{z}_{i}^{2}-\left(\frac{\delta_{i}}{2}-\frac{l_{0} l^{-1}}{2 v_{i}}\right) \underbrace{\left\|\widetilde{\theta}_{F_{i}}\right\|^{2}}_{\tilde{\theta}_{F_{i}}^{T} \widetilde{\theta}_{F_{i}}} \widetilde{\theta}_{F_{i}}]+R_{0},
\end{aligned}
$$

where $R_{0}=\sum_{i=1}^{n}\left(\left(\varrho_{i}^{2} / 2\right)+\left(\delta_{i} / 2\right)\left\|\theta_{F_{i}}^{*}\right\| s^{2}\right)$. By choosing parameters $l_{0}, l_{i}$, and $\delta_{i}$ to satisfy $c_{i}>-\left(\dot{r}_{i} / 2 r_{i}^{2}\right)$, $l_{0}>-\left(\dot{r}_{i} / 2 r_{i}^{2}\right)+\left(v_{i} l_{0} l^{-1} \chi_{i}^{2} / 2\right), \quad k_{i}>-\left(\dot{r}_{i} / 2 r_{i}^{2}\right), \quad$ and $\delta_{i}>\left(l_{0} l^{-1} / \nu_{i}\right)$, and define the following compact sets

$$
\begin{aligned}
& \Omega_{z_{i}}=\left\{z_{i}|| z_{i} \mid \leq \sqrt{\frac{R_{0}}{c_{i}+\left(\dot{r}_{i} / 2 r_{i}^{2}\right)}}\right\}, \\
& \Omega_{\widetilde{D}_{i}}=\left\{\widetilde{D}_{i}|| \widetilde{D}_{i} \mid \leq \sqrt{\frac{R_{0}}{l_{0}+\left(\dot{r}_{i} / 2 r_{i}^{2}\right)-\left(\nu_{i} l_{0} l^{-1} \chi_{i}^{2} / 2\right)}}\right\}, \\
& \Omega_{\theta_{i}}=\left\{\left\|\widetilde{\theta}_{i}\right\| \mid\left\|\widetilde{\theta}_{i}\right\| \leq \sqrt{\frac{R_{0}}{\left(\delta_{i} / 2\right)-\left(l_{0} l^{-1} / 2 \nu_{i}\right)}}\right\}, \\
& \Omega_{\widetilde{z}_{i}}=\left\{\widetilde{z}_{i}|| \widetilde{z}_{i} \mid \leq \sqrt{\frac{R_{0}}{k_{i} \pi_{i}+\left(\pi_{i} \dot{r}_{i} / 2 r_{i}^{2}\right)}}\right\} .
\end{aligned}
$$

Obviously, if $z_{i} \notin \Omega_{z_{i}}$ or $\widetilde{D}_{i} \notin \Omega_{\widetilde{D}_{i}}$ or $\left\|\widetilde{\theta}_{i}\right\| \notin \Omega_{\theta_{i}}$ or $\widetilde{z}_{i} \notin \Omega_{\widetilde{z}_{i}}, \dot{V}$ will be negative. Hence, $z_{i}, \widetilde{D}_{i},\left\|\widetilde{\theta}_{i}\right\|$, and $\widetilde{z}_{i}$ are uniformly bounded. Because $z_{i}$ is bounded, it is known from Lemma 1 that $e_{i}$ satisfies PPB (11).

Remark 4. In (22), a composite learning adaptation law for the fuzzy parameter $\widehat{\theta}$ is given. It should be noticed that this law is very important to achieve accurate approximation of unknown nonlinear functions in the PPC design. In fact, adaptive fuzzy PPC methods are used in many references, for example, in [27-30]. However, the control methods in the aforementioned literature have no such ability.

Remark 5. In the proposed method, as indicated in Theorem 1 , all signals will remain bounded. It has also been shown that the variables $z_{i}, \widetilde{D}_{i},\left\|\widetilde{\theta}_{i}\right\|$, and $\widetilde{z}_{i}$ will converge to a small region of zero whose radius is determined by control design parameters. One can adjust the radius of the region by using different parameters.

\section{Example}

In this section, Liu chaotic system [31] is introduced to show the effect of proposed control method (22), and the Liu chaotic system is described as follows:

$$
\left\{\begin{array}{l}
\dot{x}_{1}=\underbrace{-x_{1}-x_{2}^{2}}_{f_{1}(x)}+d_{1}(t)+g_{1}(x) \Gamma_{1}\left(u_{1}\right), \\
\dot{x}_{2}=\underbrace{2.5 x_{2}-4 x_{1} x_{3}}_{f_{2}(x)}+d_{2}(t)+g_{2}(x) \Gamma_{2}\left(u_{2}\right), \\
\dot{x}_{3}=\underbrace{-5 x_{3}+4 x_{1} x_{2}}_{f_{3}(x)}+d_{3}(t)+g_{3}(x) \Gamma_{3}\left(u_{3}\right),
\end{array}\right.
$$

where $g_{1}(x)=g_{2}(x)=g_{3}(x)=1, d_{1}(t)=5.5 \sin (t), d_{2}(t)=6.0$ $\sin (t)$, and $d_{3}(t)=6.5 \sin (t)$. The reference signal $\mathbf{x}_{d}=[\cos (t)$, $0.5 \cos (t),-2.6 \cos (t)]^{T}$. The state initial value is assumed unknown. In this case, the traditional PPC method cannot be applied. Assume that the tracking error is in the range $[-5$, $5]$, select the performance function $y_{1}(t)=y_{2}(t)=y_{3}(t)$ $=\operatorname{coth}(0.5 t+0.25)-1+0.1$, and initial values are chosen as $\mathbf{x}(0)=[-1,-0.5,-0.8]^{T}, \widehat{z}(0)=[1.2,1.2,1.2]^{T}, \mathbf{v}(0)=[-2,2$, $3]^{T}$, and $\widehat{\theta}_{F_{i}}(0)=0$, and parameters are chosen as $m=3, b_{r i}=b_{l i}=4, \bar{\lambda}=\underline{\lambda}=1, l_{0}=20, l_{i}=10, k_{i}=c_{i}=10$, and $\eta_{F_{i}}=\pi_{i}=3, \delta_{i}=0.5, \quad i=1,2$, 3. The fuzzy membership functions are selected as

$$
\varphi_{f}(k)=\exp \left[-\frac{1}{2}\left(\frac{k+7.5-2.5 j}{1.2}\right)^{2}\right]
$$

where $j=1,2,3,4,5 ; k=x_{1}, x_{2}, x_{3}$.

The simulation results are shown in Figures 1-6. Denote the proposed method of this paper as OBPPC. From Figures 1-3, one can see that tracking errors $e_{1}, e_{2}$, and $e_{3}$ are limited within PPB (11). Under the disturbance observer (19), $\quad \widehat{D}_{1}+l_{1}^{-1} \widehat{\theta}_{F_{1}}^{T} \varphi_{F_{1}}(\mathbf{x}), \quad \widehat{D}_{2}+l_{2}^{-1} \widehat{\theta}_{F_{2}}^{T} \varphi_{F_{2}}(\mathbf{x}), \quad$ and $\widehat{D}_{3}+$ $l_{3}^{-1} \hat{\theta}_{F_{3}}^{T} \varphi_{F_{3}}(\mathbf{x})$ can efficiently estimate $f_{1}(\mathbf{x})+d_{1}(t)+\Delta u_{1}$, $f_{2}(\mathbf{x})+d_{2}(t)+\Delta u_{2}$, and $f_{3}(\mathbf{x})+d_{3}(t)+\Delta u_{3}$ in Figure 4. Here, the existence problem of $\dot{r}_{i}$ in (32) needs to be considered. In Figure 6 , the tracking errors $e_{i}$ enter the stable region $[-0.1$, $0.1]$, but the dead-zone fluctuation of $e_{i}$ will occur due to the influence of the dead-zone inputs $u_{i}$ (see Figure 5), which will cause $r_{i}$ to fluctuate. Notice that its derivative $\dot{r}_{i}$ is bounded, and the value of $\dot{r}_{i} / 2 r_{i}^{2}$ is not larger. Therefore, this paper chooses $c_{i}=30$ which can guarantee the existence of compact sets $\Omega_{z_{i}}, \Omega_{\widetilde{D}_{i}}$, and $\Omega_{\widetilde{z}_{i}}$. 


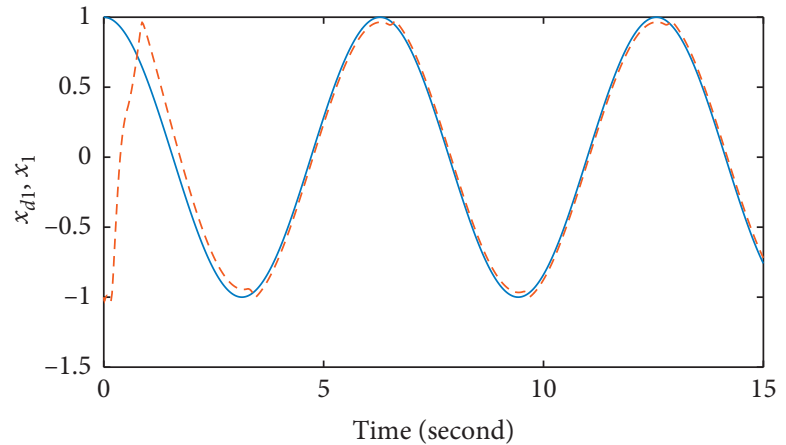

$-x_{d 1}$

$--x_{1}$ by using OBPPC

(a)

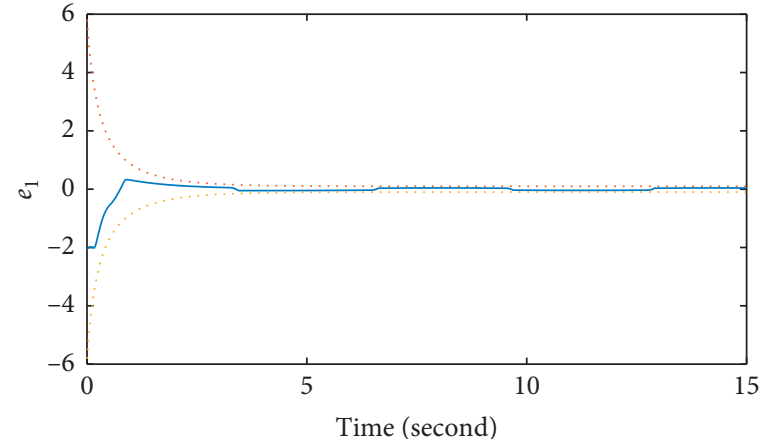

- $e_{1}$ by using OBPPC

FIgURE 1: The trajectories of (a) $x_{d 1}$ and $x_{1}$ and (b) $e_{1}$.

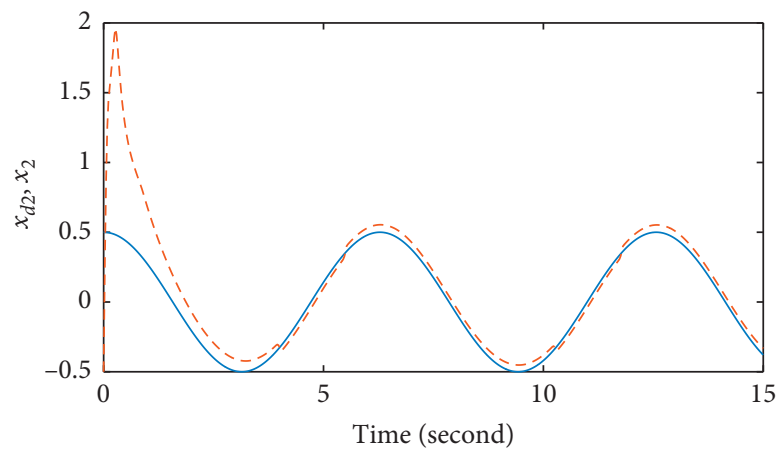

$-x_{d 2}$

$---x_{2}$ by using OBPPC

(a)

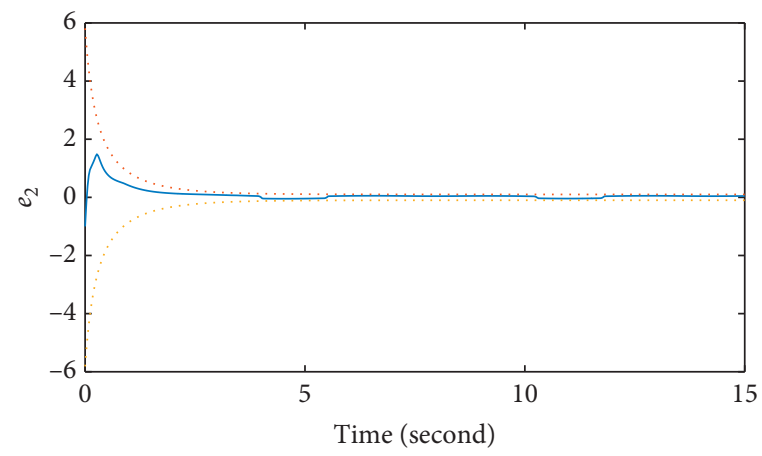

- $e_{2}$ by using OBPPC

Figure 2: The trajectories of (a) $x_{d 2}$ and $x_{2}$ and (b) $e_{2}$.

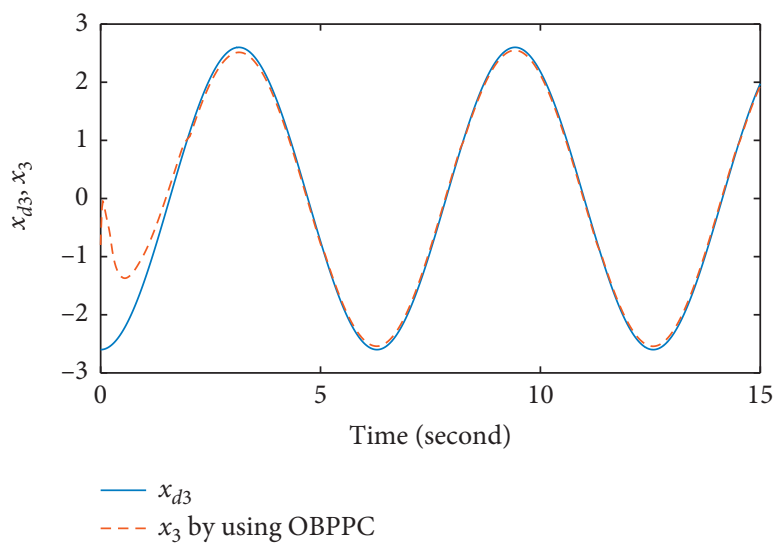

(a)

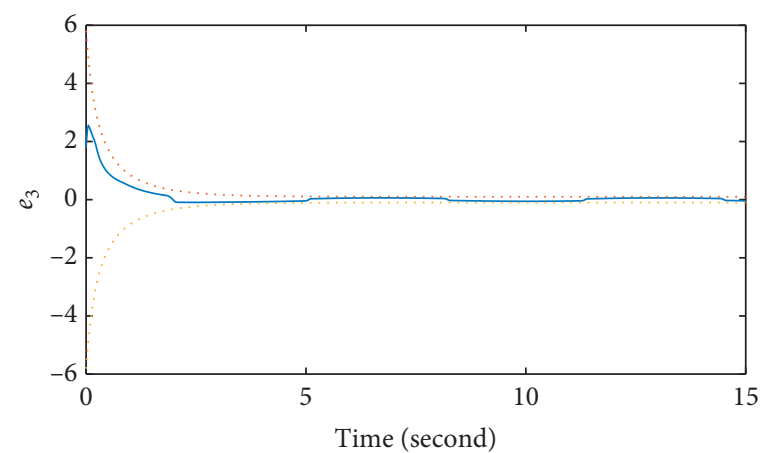

- $e_{3}$ by using OBPPC

(b)

Figure 3: The trajectories of (a) $x_{d 3}$ and $x_{3}$ and (b) $e_{3}$. 


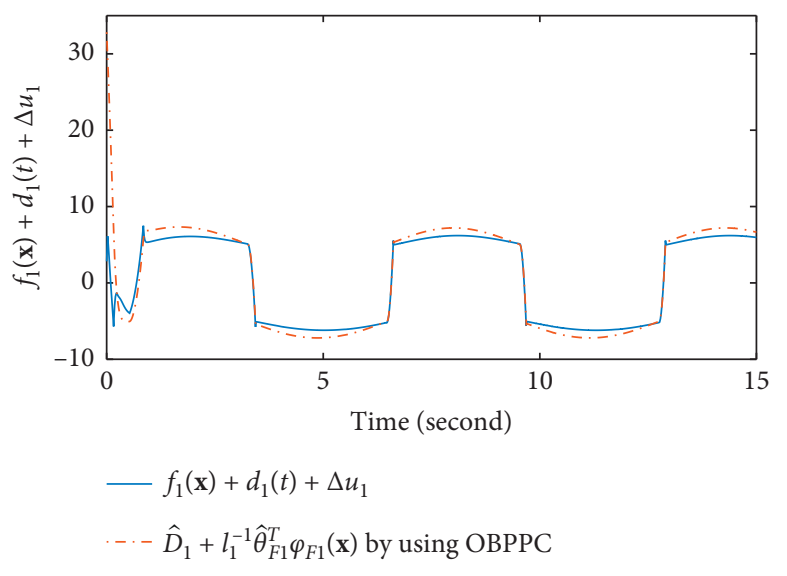

(a)

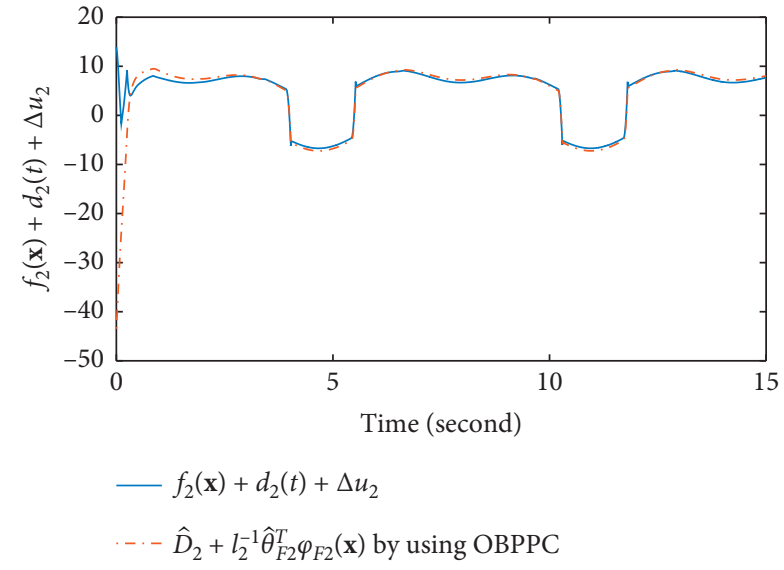

(b)

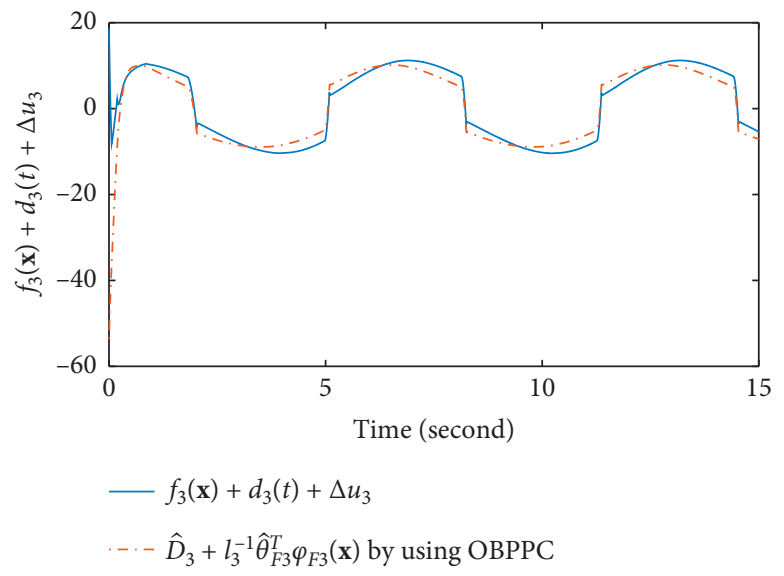

(c)

Figure 4: Estimation of (a) $f_{1}(\mathbf{x})+d_{1}(t)+\Delta u_{1}$, (b) $f_{2}(\mathbf{x})+d_{2}(t)+\Delta u_{2}$, and (c) $f_{3}(\mathbf{x})+d_{3}(t)+\Delta u_{3}$ by using OBPPC.

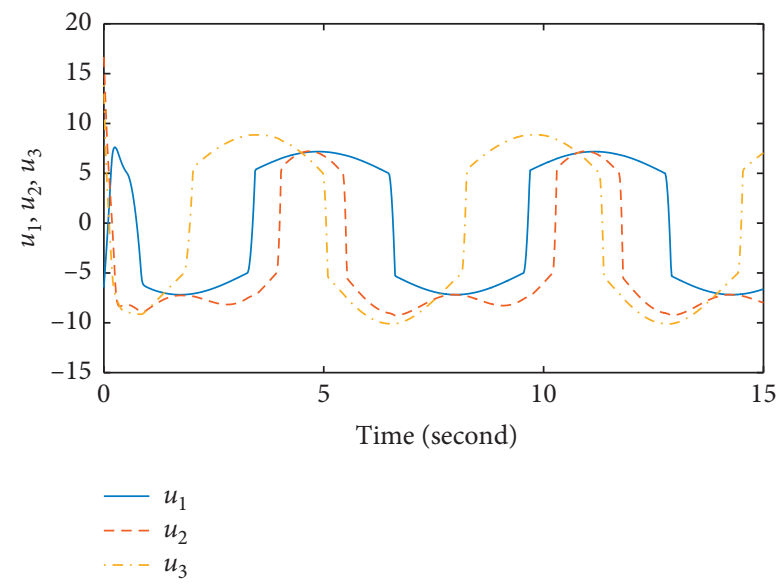

FIGURE 5: The trajectories of $u_{1}, u_{2}$, and $u_{3}$ by using OBPPC. 

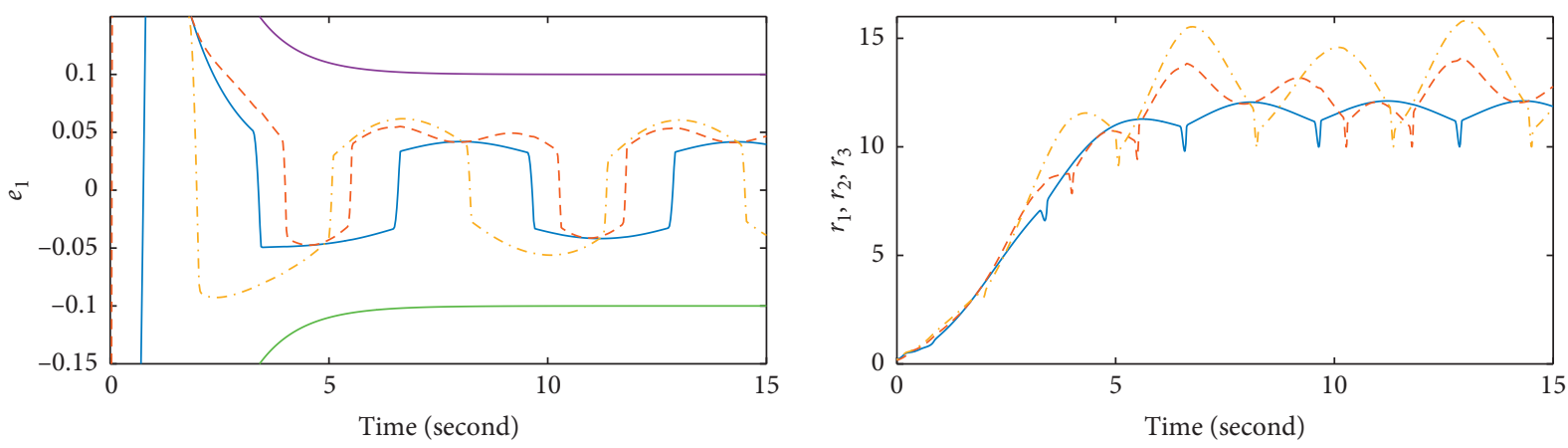

$-e_{1}, e_{2}, e_{3}$ by using TPPC

$$
\begin{aligned}
& -r_{1} \\
& ---r_{2} \\
& -.-r_{3}
\end{aligned}
$$

(a)
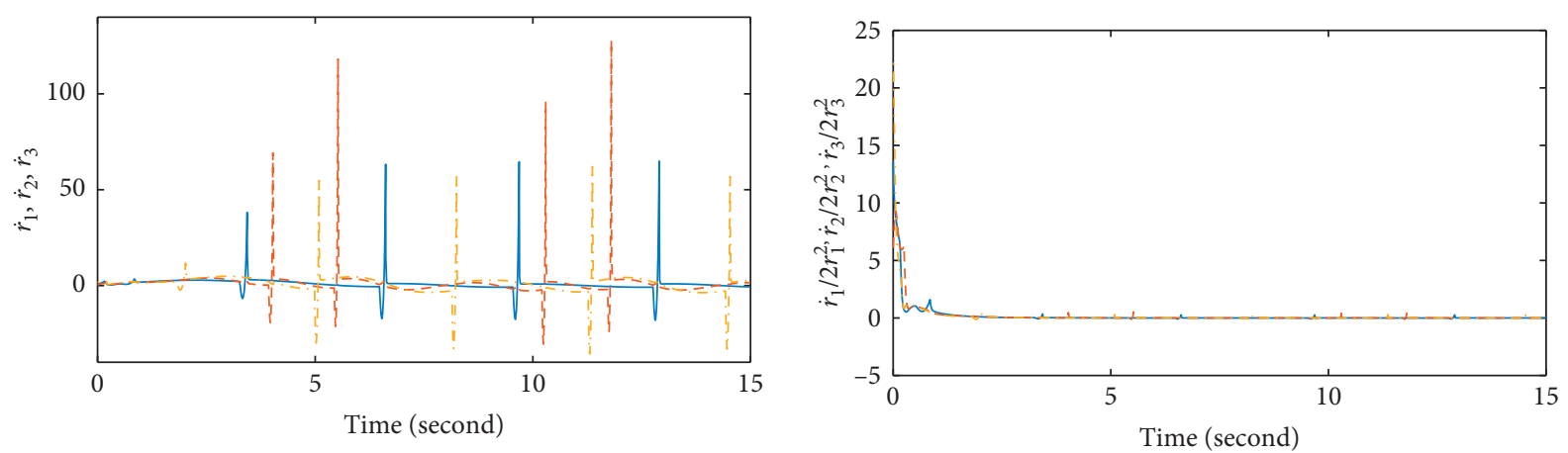

$$
\begin{aligned}
& -\dot{r}_{1} \\
& ---\dot{r}_{2} \\
& --\dot{r}_{3}
\end{aligned}
$$

$$
\begin{array}{ll} 
& \dot{r}_{1} / 2 r_{1}^{2} \\
--- & \dot{r}_{2} / 2 r_{2}^{2} \\
- & \dot{r}_{3} / 2 r_{3}^{2}
\end{array}
$$

(c)

(d)

FIGURE 6: The trajectories of (a) tracking error $e_{i}$, (b) $r_{i}$, (c) $\dot{r}_{i}$, and $(\mathrm{d}) \dot{r}_{i} / 2 r_{i}^{2}(i=1,2,3)$ by using OBPPC.

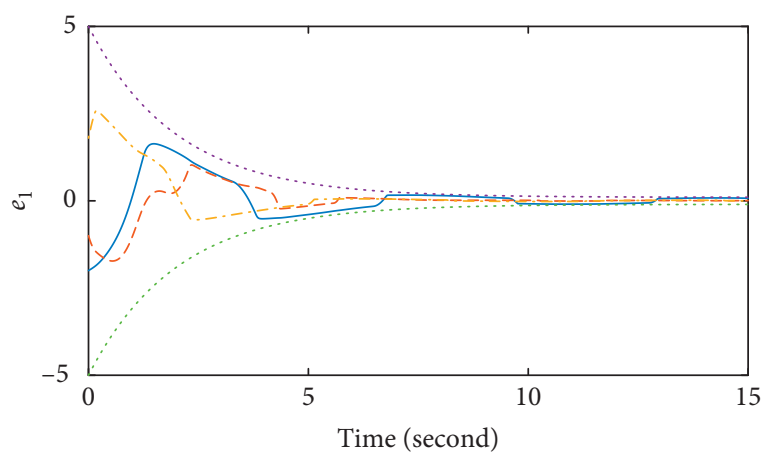

$-e_{1}, e_{2}, e_{3}$ by using TPPC

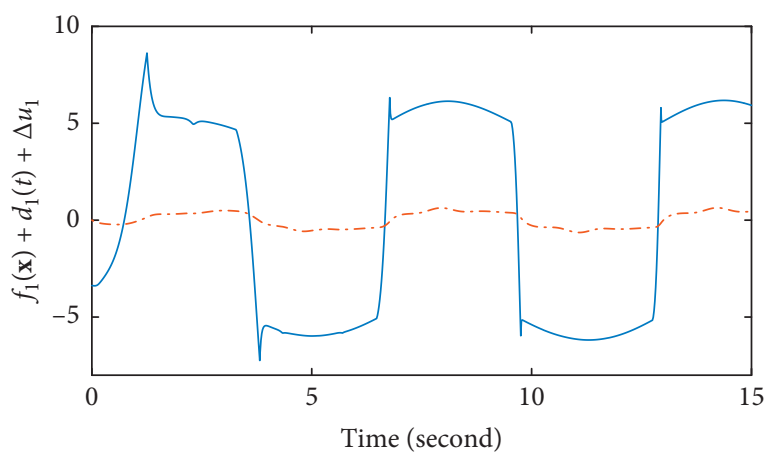

$f_{1}(\mathbf{x})+d_{1}(t)+\Delta u$ $\hat{\vartheta}_{F 1}^{T} \varphi F_{1}(\mathbf{x})$ by using TPPC

(b)

Figure 7: Continued. 


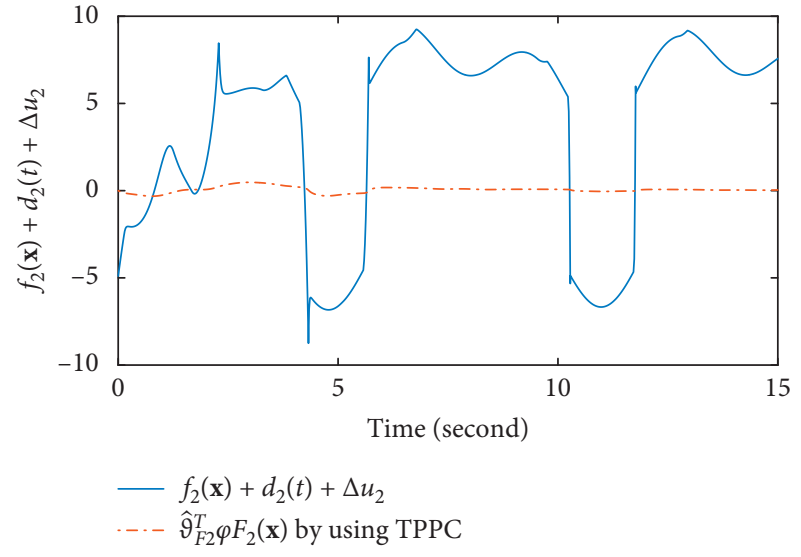

(c)

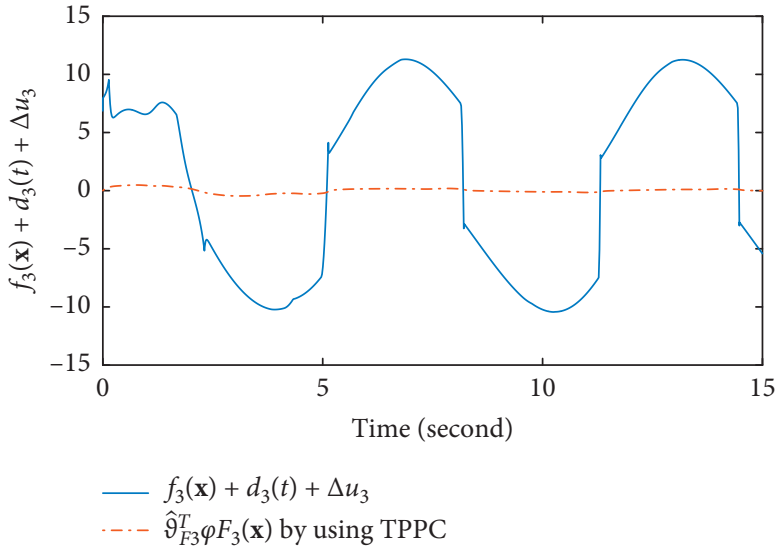

(d)

FIgURE 7: The trajectories of (a) tracking error $e_{1}, e_{2}, e_{3}$ and estimation of $(\mathrm{b}) f_{1}(\mathbf{x})+d_{1}(t)+\Delta u_{1},(\mathrm{c}) f_{2}(\mathbf{x})+d_{2}(t)+\Delta u_{2}$, and $(\mathrm{d})$ $f_{3}(\mathbf{x})+d_{3}(t)+\Delta u_{3}$ by using TPPC.

In order to compare with the traditional PPC, it is necessary to assume that the sign of the tracking error $e_{i}$ is known, and the control method law is designed as

$$
\left\{\begin{array}{l}
u_{i}=-\hat{\vartheta}_{\bar{f}_{i}}^{T} \varphi_{\bar{f}_{i}}(\mathbf{x})-30 \bar{z}_{i}+\frac{e_{i} \dot{\mu}_{i}}{\mu_{i}}+x_{d i}, \\
\dot{\hat{\vartheta}}_{\bar{f}_{i}}=40\left(\bar{z}_{1} \varphi_{\bar{f}_{i}}(\mathbf{x})-0.3 \widehat{\vartheta}_{\bar{f}_{1}}\right) \\
\bar{z}_{i}=\tanh \left(\frac{e_{i}}{\mu_{i}}\right) \\
\mu_{i}=(5.0-0.1) \exp (-5.0 t)+0.1
\end{array}\right.
$$

where $\bar{f}_{i}(\mathbf{x})=f_{i}(\mathbf{x})+d_{i}(t)+\Delta u_{i}$ and $\widehat{\vartheta}_{\bar{f}_{i}}^{T} \varphi_{\bar{f}_{i}}(\mathbf{x})$ is the estimation of $\bar{f}_{i}(\mathbf{x})$. The parameter $\lambda$ in (1) is selected as $\lambda=1$. Denote the control law (35) as TPPC. It can be seen from Figure 7 that the error $e_{i}$ satisfies (1), but the estimation of $\bar{f}_{i}(x)$ is poor. Therefore, it can be seen from the comparison that the method proposed in this paper is more effective.

\section{Conclusion}

By using the composite PPC method and the disturbance observer, this paper addresses the stability for a class of uncertain nonlinear system with unknown initial states. Firstly, a performance function is introduced to overcome the problem of unknown initial values. Then, the error system is transformed to an equivalent one by using transformation function. Through the constructed observer and fuzzy logic systems, unknown functions and timevarying external disturbances can be estimated effectively. Meanwhile, the boundedness of all involved signals is guaranteed. Moreover, the tracking errors satisfy the prescribed performance bounds all the time. How to apply the proposed method to real-world systems, such as robotic manipulators, is one of our future research directions.

\section{Data Availability}

All data generated or analyzed during this study are included in this article.

\section{Conflicts of Interest}

The authors declare that there are no conflicts of interest regarding the publication of this paper.

\section{References}

[1] S. Tong, K. Sun, and S. Sui, "Observer-based adaptive fuzzy decentralized optimal control design for strict-feedback nonlinear large-scale systems," IEEE Transactions on Fuzzy Systems, vol. 26, no. 2, pp. 569-584, 2017.

[2] H. Wang, P. X. Liu, and P. Shi, "Observer-based fuzzy adaptive output-feedback control of stochastic nonlinear multiple time-delay systems," IEEE Transactions on Cybernetics, vol. 47, no. 9, pp. 2568-2578, 2017.

[3] L. Wang, M. V. Basin, H. Li, and R. Lu, "Observer-based composite adaptive fuzzy control for nonstrict-feedback systems with actuator failures," IEEE Transactions on Fuzzy Systems, vol. 26, no. 4, pp. 2336-2347, 2017.

[4] A. M. Zaki, M. El-Bardini, F. A. S. Soliman, and M. M. Sharaf, "Embedded two level direct adaptive fuzzy controller for dc motor speed control," Ain Shams Engineering Journal, vol. 9, no. 1, pp. 65-75, 2018.

[5] Y. Pan and H. Yu, "Composite learning robot control with guaranteed parameter convergence," Automatica, vol. 89, pp. 398-406, 2018.

[6] Y. Pan, X. Li, and H. Yu, "Efficient pid tracking control of robotic manipulators driven by compliant actuators," IEEE Transactions on Control Systems Technology, vol. 27, no. 2, pp. 915-922, 2018.

[7] H. Liu, Y. Pan, S. Li, and Y. Chen, "Adaptive fuzzy backstepping control of fractional-order nonlinear systems," IEEE Transactions on Systems, Man, and Cybernetics: Systems, vol. 47, no. 8, pp. 2209-2217, 2017.

[8] H. Liu, Y. Pan, J. Cao, H. Wang, and Y. Zhou, "Adaptive neural network backstepping control of fractionial-order 
nonlinear systems with actuator faults," IEEE Transactions on Neural Networks and Learning Systems, 2020.

[9] H. Liu, Y. Pan, J. Cao, Y. Zhou, and H. Wang, "Positivity and stability analysis for fractional-order delayed systems: a T-S fuzzy model approach," IEEE Transactions on Fuzzy Systems, 2020.

[10] A. E. Matouk, "Chaos synchronization of a fractional-order modified Van der pol-duffing system via new linear control, backstepping control and Takagi-Sugeno fuzzy approaches," Complexity, vol. 21, no. 1, pp. 116-124, 2016.

[11] Q. Zhong, J. Bao, Y. Yu, and X. Liao, "Impulsive control for fractional order chaotic system," Chinese Physics Letters, vol. 25, no. 8, p. 2812, 2008.

[12] C. P. Bechlioulis and G. A. Rovithakis, "Robust adaptive control of feedback linearizable mimo nonlinear systems with prescribed performance," IEEE Transactions on Automatic Control, vol. 53, no. 9, pp. 2090-2099, 2008.

[13] Y. Li, X. Shao, and S. Tong, "Adaptive fuzzy prescribed performance control of non-triangular structure nonlinear systems," IEEE Transactions on Fuzzy Systems, 2019.

[14] X. Shao and S. Tong, "Adaptive fuzzy prescribed performance control for mimo stochastic nonlinear systems," IEEE Access, vol. 6, pp. 76754-76767, 2018.

[15] C. P. Bechlioulis and G. A. Rovithakis, "Robust partial-state feedback prescribed performance control of cascade systems with unknown nonlinearities," IEEE Transactions on Automatic Control, vol. 56, no. 9, pp. 2224-2230, 2011.

[16] C. P. Bechlioulis and G. A. Rovithakis, "A priori guaranteed evolution within the neural network approximation set and robustness expansion via prescribed performance control," IEEE Transactions on Neural Networks and Learning Systems, vol. 23, no. 4, pp. 669-675, 2012.

[17] X. Bu, X. Wu, F. Zhu, J. Huang, Z. Ma, and R. Zhang, "Novel prescribed performance neural control of a flexible airbreathing hypersonic vehicle with unknown initial errors," ISA Transactions, vol. 59, pp. 149-159, 2015.

[18] H.-S. Ahn, Y. Chen, and K. L. Moore, "Iterative learning control: brief survey and categorization," IEEE Transactions on Systems, Man and Cybernetics, Part C (Applications and Reviews), vol. 37, no. 6, pp. 1099-1121, 2007.

[19] W. He, T. Meng, X. He, and S. S. Ge, "Unified iterative learning control for flexible structures with input constraints," Automatica, vol. 96, pp. 326-336, 2018.

[20] D. Meng and K. L. Moore, "Robust iterative learning control for nonrepetitive uncertain systems," IEEE Transactions on Automatic Control, vol. 62, no. 2, pp. 907-913, 2017.

[21] Y. Pan and H. Yu, "Composite learning from adaptive dynamic surface control," IEEE Transactions on Automatic Control, vol. 61, no. 9, pp. 2603-2609, 2016.

[22] Y. Pan, T. Sun, and H. Yu, "Composite adaptive dynamic surface control using online recorded data," International Journal of Robust and Nonlinear Control, vol. 26, no. 18, pp. 3921-3936, 2016.

[23] B. Xu, F. Sun, Y. Pan, and B. Chen, "Disturbance observer based composite learning fuzzy control of nonlinear systems with unknown dead zone," IEEE Transactions on Systems, Man, and Cybernetics: Systems, vol. 47, no. 8, pp. 1854-1862, 2017.

[24] B. Xu, Z. Shi, and C. Yang, "Composite fuzzy control of a class of uncertain nonlinear systems with disturbance observer," Nonlinear Dynamics, vol. 80, no. 1-2, pp. 341-351, 2015.

[25] H. Liu, Y. Pan, and J. Cao, "Composite learning adaptive dynamic surface control of fractional-order nonlinear systems," IEEE Transactions on Cybernetics, 2019.
[26] H. Liu, H. Wang, J. Cao, A. Alsaedi, and T. Hayat, "Composite learning adaptive sliding mode control of fractional-order nonlinear systems with actuator faults," Journal of the Franklin Institute, vol. 356, no. 16, pp. 9580-9599, 2019.

[27] Q. Zhou, H. Li, L. Wang, and R. Lu, "Prescribed performance observer-based adaptive fuzzy control for nonstrict-feedback stochastic nonlinear systems," IEEE Transactions on Systems, Man, and Cybernetics: Systems, vol. 48, no. 10, pp. 1747-1758, 2017.

[28] S. Sui, S. Tong, and Y. Li, "Observer-based fuzzy adaptive prescribed performance tracking control for nonlinear stochastic systems with input saturation," Neurocomputing, vol. 158, pp. 100-108, 2015.

[29] J. Qiu, K. Sun, T. Wang, and H. Gao, "Observer-based fuzzy adaptive event-triggered control for pure-feedback nonlinear systems with prescribed performance," IEEE Transactions on Fuzzy Systems, vol. 27, no. 11, 2019.

[30] Y. Liu, X. Liu, and Y. Jing, "Adaptive fuzzy finite-time stability of uncertain nonlinear systems based on prescribed performance," Fuzzy Sets and Systems, vol. 374, pp. 23-39, 2019.

[31] M. T. Yassen, "Controlling, synchronization and tracking chaotic liu system using active backstepping design," Physics Letters A, vol. 360, no. 4-5, pp. 582-587, 2007. 